\title{
Efficient one-pot synthesis of 2-oxazolines from benzoylacetonitrile and $\beta$-aminoalcohols mediated by $\mathrm{ZnCl}_{2}$
}

\author{
MEI LUO ${ }^{\mathrm{a}, *}$, JING CHENG ZHANG ${ }^{\mathrm{a}}$ and HAO YIN ${ }^{\mathrm{b}}$ \\ ${ }^{a}$ Hefei University of Technology, Hefei, Anhui, China, 230009 \\ ${ }^{b}$ University of Science and Technology of China, Hefei, 230009, China \\ e-mail: luomei@pku.edu.cn
}

MS received 10 April 2014; revised 06 July 2014; accepted 09 July 2014

\begin{abstract}
A series of 2-oxazolines were synthesized using a simple, one-pot method under inert, moisturefree conditions from the benzoylacetonitrile and $\beta$-aminoalcohols mediated by $115-172 \mathrm{~mol}^{2} \mathrm{ZnCl}_{2}$. Structures of products were fully characterized by NMR, IR and MS.
\end{abstract}

Keywords. One-pot; benzoylacetonitrile; $\beta$-aminoalcohols; $\mathrm{ZnCl}_{2}$

\section{Introduction}

2-Oxazolines and their organometallic complexes were used as catalysts in organic reactions and polymerizations. ${ }^{1}$ The reported methods for synthesis of 2 -oxazolines include the following: direct condensationcyclodehydration of carboxylic acids with amino alcohols $;{ }^{2}$ mediation by $\mathrm{N}, \mathrm{N}$ '-diisopropylcarbodiimide; ${ }^{3}$ use of aldehydes, amino alcohols, or $\mathrm{N}$-bromosuccinimide as an oxidizing agent ${ }^{4}$ reaction of nitriles with $\beta$-amino alcohols under thermal, ultrasonic or microwave irradiation using $\mathrm{InCl}_{3}$ as a catalyst; ${ }^{5}$ and tungstophosphoric acid-catalyzed formation. ${ }^{6}$ The one-pot synthesis is characterized by mild reaction conditions, broad scope, high yields and preparative simplicity. Encouraged by the pioneering work, we wish to report the novel methodology for the synthesis of 2-oxazolines from the benzoylacetonitrile and $\beta$-aminoalcohols using harsh reaction conditions (115-172 $\mathrm{mol} \% \mathrm{ZnCl} 2)$.

\section{Experimental}

Benzoylacetonitrile and amino alcohol were purchased from Acros, Aldrich, Fluka. Flash column chromatography was performed using E. Merck silica gel (60, particle size $0.02-0.03 \mathrm{~mm}),{ }^{1} \mathrm{H}$ and ${ }^{13} \mathrm{C}$ NMR and ${ }^{31} \mathrm{P}$ NMR spectra were obtained using Bruker AM500 spectrometer. Proton chemical shifts are reported in ppm $(\delta)$ with the solvent relative to tetramethylsilane (TMS) employed as the internal standard $\left(\mathrm{CDCl}_{3}, \delta\right.$ $7.26 \mathrm{ppm})$. The following abbreviations were used to designate chemical shift mutiplicities: $\mathrm{s}=$ singlet,

\footnotetext{
*For correspondence
}

$\mathrm{d}=$ doublet, $\mathrm{t}=$ triplet, $\mathrm{m}=$ multiplet. Infrared spectra were recorded on a Mattson Galaxy Series FTIR 3000 spectrometer; peaks are reported in $\mathrm{cm}^{-1}$. Elemental analysis were obtained on Elemental Analyzer AE-3000, High resolution mass spectra (HRMS) were obtained on Micro GCT-MS equipped with an EI ion source. Optical rotations were measured on WZZ-1 automatic polarimeter with a $2 \mathrm{~cm}$ cell at the sodium D-line.

\subsection{Structure determination}

The yellow prismatic crystal of the intermediate $\mathbf{3 a}$ of approximately $0.156 \times 0.132 \times 0.034 \mathrm{~mm}$ was selected for the data collection on a 'graphite' diffractometer with mirror monochromated $\mathrm{MoK} / \alpha$ radiation $(\lambda=0.71073 \AA)$. A total of 4911 reflections were collected in the range of $2.29<\theta<25.99^{\circ}$ by using 'phi and omega scans' techniques at 293(2) $\mathrm{K}, \mathrm{C}_{15} \mathrm{H}_{20} \mathrm{~N}_{2} \mathrm{O}$, $M=244.33$, monoclinic, C $2, \mathrm{a}=8.794(4) \AA, \alpha=90^{\circ}$, $\mathrm{b}=9.092(4) \AA, \beta=113.290(7)^{\circ}, \mathrm{c}=9.686(5) \AA$, $\gamma=90^{\circ}, V=711.3(6) \AA^{3}, \mathrm{Z}=2, \mathrm{D}_{\text {calc. }}=1.141 \mathrm{mg} / \mathrm{m}^{3}$, the final $\mathrm{R}$ factor was $\mathrm{R}_{1}=0.0633,2770$ for reflections with $I_{0}>2 \sigma\left(I_{0}\right), \mathrm{R}_{\omega}=0.639$ for all data. The structure were solved by full-matrix least-squares on $\mathrm{F}^{2}$ using the SHELXTL PROGREM.,8

The yellow prismatic crystal of the intermediate 5c of approximately $0.28 \times 0.08 \times 0.04 \mathrm{~mm}$ was selected for the data collection on a 'graphite' diffractometer with mirror monochromated Mo $\mathrm{K} / \alpha$ radiation $(\lambda=0.71073 \AA)$. A total of 4194 reflections were collected in the range of $0.9969<\theta<0.9784^{\circ}$ by using 'phi and omega scans' techniques at 293(2) $\mathrm{K}, \mathrm{C}_{15} \mathrm{H}_{13}$ NO, $M=223.26$, monoclinic, P2(1), a = 10.235(4) $\mathrm{A}$, $\alpha=90^{\circ}, \mathrm{b}=5.846(2) \AA, \beta=110.355(6)^{\circ}, \mathrm{c}=$ 
10.614(4) $\AA, \gamma=90^{\circ}, V=595.3(4) \AA^{3}, \mathrm{Z}=2, \mathrm{D}_{\text {calc. }}=$ $1.245 \mathrm{mg} / \mathrm{m}^{3}$, the final $\mathrm{R}$ factor was $\mathrm{R}_{1}=0.0302$, 2280 for reflections with $I_{0}>2 \sigma\left(I_{0}\right), \mathrm{R}_{\omega}=0.0485$ for all data. The structure were solved by full-matrix least-squares on $\mathrm{F}^{2}$ using the SHELXTL PROGREM. ${ }^{7,8}$

2.2 The synthesis of (4R/S)-4,5-dihydro-4-(R group $)-2-$ phenyloxazole $(\mathbf{4 a - 4 b}$ and $\mathbf{5 c - 5 d})$

Dry $\mathrm{ZnCl}_{2}(8.29-12.41 \mathrm{mmol})$ benzoylacetonitrile $(1.0 \mathrm{~g})$ and L-amino alcohol (4.2-4.8g) were combined under anhydrous and oxygen-free conditions in a dry 100 $\mathrm{mL}$ Schlenk flask. The reagents were dissolved in 50 $\mathrm{mL}$ of dry chlorobenzene and the reaction mixture was refluxed for $72 \mathrm{~h}$. The solvent was removed under reduced pressure, and the resulting residue was dissolved in $15 \mathrm{~mL} \mathrm{H}_{2} \mathrm{O}$, extracted with dichloromethane $(20 \times 3 \mathrm{~mL})$. The solvent was removed under vacuum to afford crude red oil. Further purification was achieved using silica gel (petroleum ether/dichlormethane 3/7). The solvent was slowly evaporated from the last fraction collected to yield colourless crystals.

2.2a (4a): The same procedure as described 2.2. The colourless crystals, M.p. $108-112^{\circ} \mathrm{C}$, yield: $65 \%$; $[\mathrm{a}]_{\mathrm{D}}^{5}=-80.0^{\circ}\left(\mathrm{c}=0.23, \mathrm{CH}_{2} \mathrm{Cl}_{2}\right):{ }^{1} \mathrm{H}$ NMR $(600 \mathrm{MHz}$, $\left.\mathrm{CDCl}_{3}, 27^{\circ} \mathrm{C}\right), \delta(\mathrm{ppm})=7.92(\mathrm{~d}, J=6.6 \mathrm{~Hz}, 2 \mathrm{H})$, 7.37 7.44(m, 3H), 4.46 4.49(m, 1H), 4.30 4.32(m, $1 \mathrm{H}), 3.97(\mathrm{t}, J=0.6 \mathrm{~Hz}, 1 \mathrm{H}), 1.78 \sim 1.83(\mathrm{~m}, 1 \mathrm{H})$, $1.68 \sim 1.72(\mathrm{~m}, 1 \mathrm{H}), 1.34 \sim 1.39(\mathrm{~m}, 1 \mathrm{H}), 0.95 \sim 0.97(\mathrm{dd}$, $J=6.6 \mathrm{~Hz}, 6.6 \mathrm{~Hz}, 6 \mathrm{H}),{ }^{13} \mathrm{C} \mathrm{NMR}\left(150 \mathrm{MHz}, \mathrm{CDCl}_{3}\right.$, $\left.27^{\circ} \mathrm{C}\right) 163.2,131.1(\mathrm{x} 2), 128.2(\mathrm{x} 4), 73.1,65.1,45.6$, 25.5, 22.9, 22.7; IR (KBr), $\mathrm{cm}^{-1}: 3435,3062,2957$,
2927, 2870, 1652, 1580, 1495, 1468, 1450, 1358, 1327, 1310, 1276, 1174, 1079, 1063, 1026, 1195, 972, 945, 779, 695; HRMS(EI):m/z (\%): calcd for $\mathrm{C}_{13} \mathrm{H}_{17} \mathrm{NO}$ : 203.1310; found: 203.1299 .

$2.2 \mathrm{~b} \quad(4 b)$ : The same procedure as described 2.2. The colourless crystals, yield: $68 \%$; M.p. $98-102^{\circ} \mathrm{C}[\mathrm{a}]_{\mathrm{D}}^{5}=$ $-65.7^{\circ}\left(\mathrm{c}=0.024, \mathrm{CH}_{2} \mathrm{Cl}_{2}\right):{ }^{1} \mathrm{H}$ NMR $(600 \mathrm{MHz}$, $\left.\mathrm{CDCl}_{3}, 27^{\circ} \mathrm{C}\right), \delta(\mathrm{ppm})=7.98(\mathrm{~d}, J=6 \mathrm{~Hz} \sim 2 \mathrm{H})$, 7.40 7.46 (m, 3H), 4.40 4.41 (m, 1H), 4.10-4.14 (m, $2 \mathrm{H}), 1.85-1.88(\mathrm{~m}, 1 \mathrm{H}), 0.93 \sim 1.05(\mathrm{dd}, J=5.5 \mathrm{~Hz}$, $5.5 \mathrm{~Hz}, 6 \mathrm{H}),{ }^{13} \mathrm{C} \mathrm{NMR}\left(150 \mathrm{MHz}, \mathrm{CDCl}_{3}, 27^{\circ} \mathrm{C}\right) 163.2$, 131.1(x2), 128.1(x4), 72.5, 69.9, 32.7, 18.8, 18.0.; IR $(\mathrm{KBr}) \mathrm{cm}^{-1}: 2959,2930,2898,2873,1652,1580,1495$, 1467, 1450, 1385, 1354, 1318, 1305, 1286, 1081, 1065, 1026, 968, 904, 780, 694; HRMS(EI):m/z (\%): calcd for $\mathrm{C}_{12} \mathrm{H}_{15} \mathrm{NO}$ : 189.1154; found: 189.1152 .

2.2c (5c): The same procedure as described 2.2. The colourless crystals, yield: $70 \%$; M.p. $134-138^{\circ} \mathrm{C}$, $[\mathrm{a}]_{\mathrm{D}}^{5}=+27.7^{\circ}\left(\mathrm{c}=0.087, \mathrm{CH}_{2} \mathrm{Cl}_{2}\right):{ }^{1} \mathrm{H} \mathrm{NMR}(500 \mathrm{MHz}$, $\left.\mathrm{CDCl}_{3}, 27^{\circ} \mathrm{C}\right), \delta(\mathrm{ppm})=8.14(\mathrm{~d}, \mathrm{~J}=5.5 \mathrm{~Hz}, 2 \mathrm{H})$, $7.34 \sim 7.55(\mathrm{~m}, 8 \mathrm{H}), 5.43 \sim 5.44(\mathrm{~m}, 1 \mathrm{H}), 4.80 \sim 4.82(\mathrm{~m}$, $1 \mathrm{H}), 4.29 \sim 4.32(\mathrm{~m}, 1 \mathrm{H}) ;{ }^{13} \mathrm{C} \mathrm{NMR}\left(125 \mathrm{MHz}, \mathrm{CDCl}_{3}\right.$, $\left.27^{\circ} \mathrm{C}\right) 164.7,142.4,131.6(\mathrm{x} 2), 128.8(\mathrm{x} 2) 128.5(\mathrm{x} 2)$, 128.4(x2), 127.6(x2), 126.8, 74.9, 70.2; IR (KBr) $\mathrm{cm}^{-1}:$, 3062, 3030, 2965, 2898, 1647, 1603, 1580, $1495,1473,1450,1357,1354,1317,1300,1277$, 1246, 1084, 1066, 1025, 974, 952, 894, 780, 760, 696, 678, 539; HRMS(EI): m/z (\%): calcd for $\mathrm{C}_{15} \mathrm{H}_{13} \mathrm{NO}$ : 223.0997; found: 223.0994 .

$2.2 \mathrm{~d}(\mathbf{5} \boldsymbol{d})$ : The same procedure as described 2.2. The colourless crystals, yield: $72 \%$, M.p. $140-144^{\circ} \mathrm{C}$

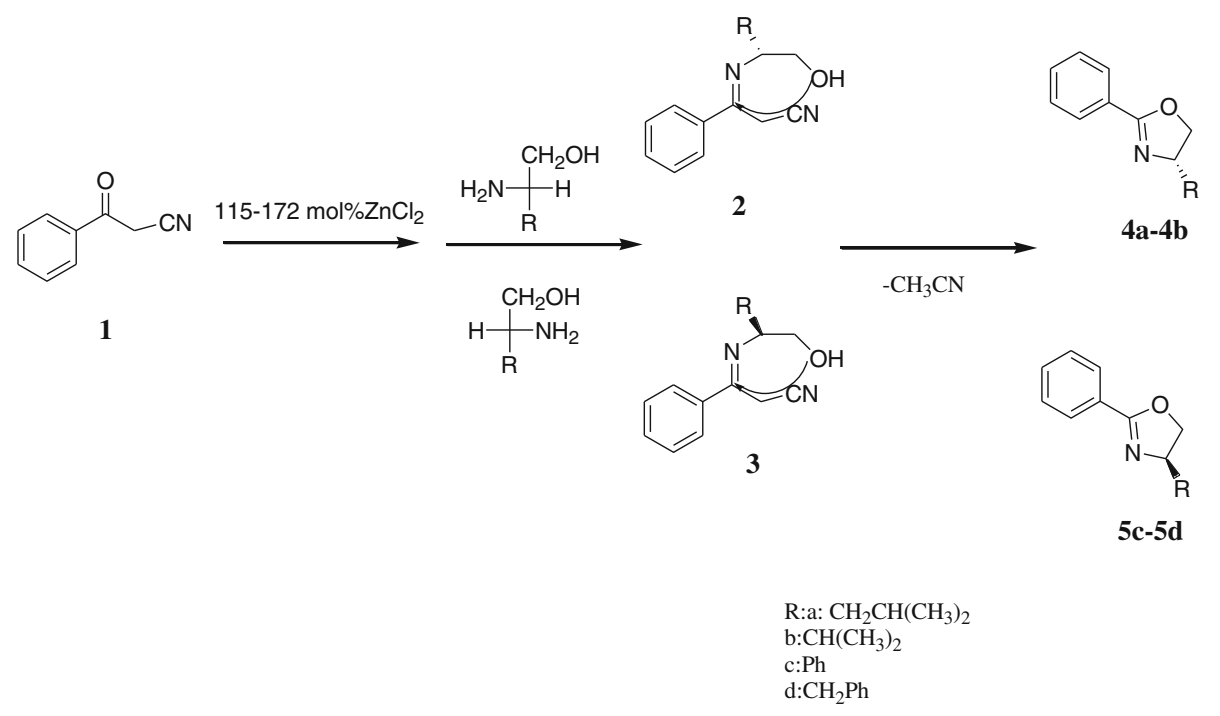

Scheme 1. The synthetic routes to the compounds $\mathbf{4}$ and $\mathbf{5}$ 
$[\mathrm{a}]_{\mathrm{D}}^{5}=+36.2^{\circ}\left(\mathrm{c}=0.30, \mathrm{CH}_{2} \mathrm{Cl}_{2}\right):{ }^{1} \mathrm{H} \mathrm{NMR}(500 \mathrm{MHz}$, $\left.\mathrm{CDCl}_{3}, 27^{\circ} \mathrm{C}\right), \delta(\mathrm{ppm})=8.0(\mathrm{t}, J=0.6 \mathrm{~Hz}, 2 \mathrm{H})$, $7.23 \sim 7.48(\mathrm{~m}, 7 \mathrm{H}), 4.56 \sim 4.61(\mathrm{~m}, 1 \mathrm{H}), 4.32(\mathrm{t}, 1 \mathrm{H})$, $4.13(\mathrm{t}, J=0.6 \mathrm{~Hz}, 1 \mathrm{H}) ; 3.24 \sim 3.27(\mathrm{dd}, J=0.54 \mathrm{~Hz}$, $0.66 \mathrm{~Hz}, 1 \mathrm{H}), 2.72 \sim 2.76(\mathrm{dd}, J=0.64 \mathrm{~Hz}, 0.8 \mathrm{~Hz}, 2 \mathrm{H})$, ${ }^{13} \mathrm{C} \mathrm{NMR}\left(125 \mathrm{MHz}, \mathrm{CDCl}_{3}, 27^{\circ} \mathrm{C}\right) 163.7,137.8,131.2$ 129.1(x3) 128.4(x3),128.1(x2),126.3(x2), 71.6, 67.6, 41.6; IR(KBr) $\mathrm{cm}^{-1}$ : 3061, 3028, 2924, 2897, 1651, 1603, 1580, 1495, 1451, 1357, 1281, 1084, 1060, 1025, 968, 780, 696, 678; HRMS(EI): m/z (\%): calcd for $\mathrm{C}_{16} \mathrm{H}_{15} \mathrm{NO}$ : 237.1154; found: 237.1150.

\section{3 (E)-[1(R)-Hydroxymethyl-3-methyl-butylimino]-3- phenyl-propionitrile (intermediate $3 a$ )}

$1.2361 \mathrm{~g}(9.09 \mathrm{mmol})$ of dry $\mathrm{ZnCl}_{2}$, benzoylacetonitrile $1.0057 \mathrm{~g}(6.93 \mathrm{mmol})$ and L-Leucinol $4.7627 \mathrm{~g}$ were added under free-water and free-oxygen condition in a dry $100 \mathrm{~mL}$ Schlenk flask. They were dissolved in $50 \mathrm{~mL}$ of dry chlorobenzene, the reaction mixture was refluxed for $72 \mathrm{~h}$. The solvent was removed under reduced pressure and the residue was dissolved in 15 $\mathrm{mL} \mathrm{H}_{2} \mathrm{O}$, extracted with $20 \times 3 \mathrm{~mL}$ of dichloromethane, the solvent was removed under vacuum, gave the crude red oil. Further purification was performed by silica gel (petroleum ether/dichlormethane 3/7), then slowly evaporation from the collected last component to give a colourless crystals, yield: $40 \%$; $[\mathrm{a}]_{\mathrm{D}}^{20}=-54.5^{\circ}(\mathrm{c}=$ 0.011, $\left.\mathrm{CH}_{3} \mathrm{OH}\right):{ }^{1} \mathrm{H}$ NMR $\left(600 \mathrm{MHz}, \mathrm{CDCl}_{3}, 27^{\circ} \mathrm{C}\right), \delta$ $(\mathrm{ppm})=7.43 \sim 7.46(\mathrm{~m}, 5 \mathrm{H}), 6.68(\mathrm{~d}, J=0.60 \mathrm{~Hz}$, $1 \mathrm{H}), 4.73 \sim 4.74(\mathrm{~m}, 1 \mathrm{H}), 4.14(\mathrm{t}, J=1 \mathrm{~Hz}, 1 \mathrm{H}), 3.30$ $(\mathrm{s}, 1 \mathrm{H}), 2.47(\mathrm{t}, J=0 \mathrm{~Hz}, 2 \mathrm{H}), 1.66 \sim 1.68(\mathrm{~m}, 1 \mathrm{H})$, $1.36 \sim 1.42(\mathrm{~m}, 2 \mathrm{H}), 0.84 \sim 1.32(\mathrm{~m}, 6 \mathrm{H}) ;{ }^{13} \mathrm{C} \mathrm{NMR}$ $\left(150 \mathrm{MHz}, \mathrm{CDCl}_{3}, 27^{\circ} \mathrm{C}\right) 157.1,130.7,124.3,122.8$ (x2), 122.7(x2), 117.1, 57.2, 52.0, 48.1, 19.0, 17.7(x2), 16.6; IR (KBr) cm $\mathrm{cm}^{-1}: 3360,3259,3072,2961,2934$, 2910, 2875, 2194, 1590, 1571, 1548, 1469, 1370, 1308,
1286, 1059, 1035, 777, 696, 659; HRMS(EI):m/z (\%): calcd for $\mathrm{C}_{15} \mathrm{H}_{20} \mathrm{~N}_{2} \mathrm{O}$ : 244.1576; found: 244.1572.

\section{Results and Discussion}

\subsection{Synthesis}

2-Oxazolines $\mathbf{4}$ and $\mathbf{5}$ were obtained in moderate yields (30-45\%) using chlorobenzene under dry, anaerobic conditions. Our first goal was to obtain the novel oxazolinyl-zinc complexes when using a large amount of Lewis acid, and up to 140-172 mol\% was used. The synthetic routes are shown in scheme 1.

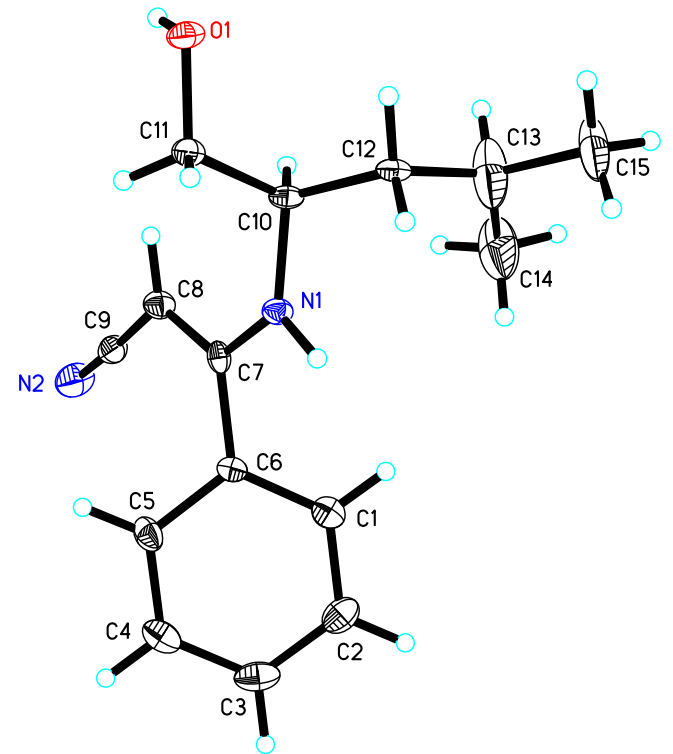

Figure 2. The crystal structure of the intermediate $3 \mathrm{a}$. Selected bond length $(\AA)$ : N(1)-C(7)1.357(4); N(1)-C(10) 1.455(4); N(2)-C(9)1.152(5); O(1)-C(11)1.423(4). Selected bond angels( $\left(^{\circ}\right): \mathrm{C}(7)-\mathrm{N}(1)-\mathrm{C}(10) 125.1(3) ; \mathrm{N}(1)-\mathrm{C}(7)-\mathrm{C}(8)$ 123.2(3); N(1)-C(7)-C(6) 114.4(3); N(2)-C(9)-C(8)176.8(4); $\mathrm{N}(1)-\mathrm{C}(10)-\mathrm{C}(11) 109.8(3) ; \mathrm{N}(1)-\mathrm{C}(10)-\mathrm{C}(12) 108.9(3)$.

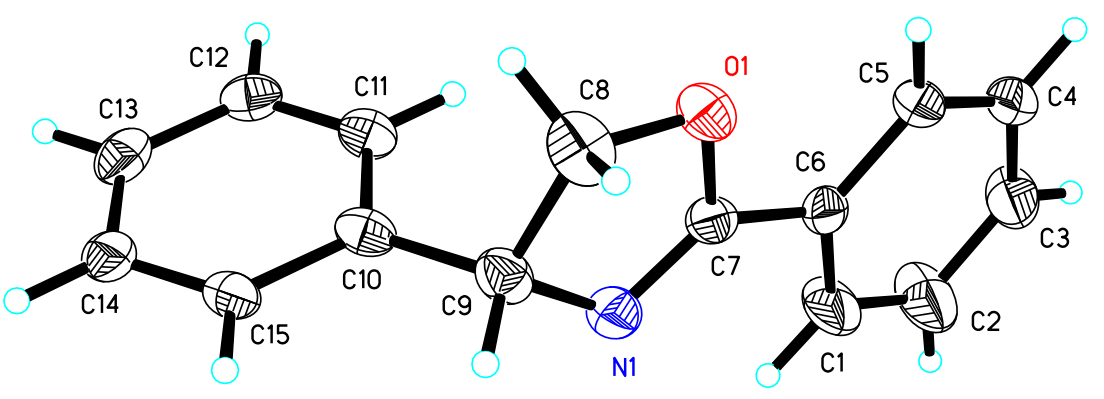

Figure 1. The crystal structure of the compound 5c. Selected bond length $(\AA)$ : $\mathrm{O}(1)-\mathrm{C}(7) 1.368(3)$; O(1)-C(8)1.445(4); N(1)-C(7)1.272(3) N(1)-C(9)1.504(4). Selected bond angels $\left(^{\circ}\right)$ : C(7)-O(1)-C(8)105.6(2); C(7)-N(1)-C(9)107.1(2); $\mathrm{C}(2)-\mathrm{C}(1)-\mathrm{C}(6) 120.1(3)$. 
On the basis of the results described above, the mechanism of formation of chiral compounds $\mathbf{4}$ and 5 can be proposed as nucleophilic attack onto $\mathrm{ZnCl}_{2}$ coordinated benzoylacetonitrile followed by cyclization and removal of acetonitrile group.

\subsection{Structure}

The crystal structures of $\mathbf{3 a}$ and $\mathbf{5 c}$ were obtained after isolation of the compounds from the solvent used for column chromatography, $\mathrm{CH}_{2} \mathrm{Cl}_{2}$ /petroleum ether. C$\mathrm{N}, \mathrm{C}=\mathrm{N}$ and $\mathrm{C} \equiv \mathrm{N}$ bonds were undoubtedly formed [C-N 1.504(4) $\AA(3 \mathrm{a}), 1.455(4) \AA(4 \mathrm{~b}) ; \mathrm{C}=\mathrm{N} 1.357(4) \AA$ (3a), $1.299 \AA$ (4b); $\mathrm{C} \equiv \mathrm{N} 1.152 \AA$ (3a)], suggesting that 4 is an intermediate in the formation of 2-oxazolines (figures 1 and 2).

\section{Conclusions}

We have shown that a large amount of $\mathrm{ZnCl}_{2}$ effectively promotes one-pot synthesis of oxazoline. This reaction is advantageous because it does not require a strong alkaline medium and uses the inexpensive catalyst $\mathrm{ZnCl}_{2}$.

Further studies are in progress to examine the scope of the one-pot synthesis of chiral organometallic complexes.

\section{Supplementary Information}

Full experimental detail, ${ }^{1} \mathrm{H}$ and ${ }^{13} \mathrm{C}$ NMR spectra. This material can be found at www.ias.ac.in/chemsci. Crystallographic data for the structure $\mathbf{4 c}$ and $\mathbf{3 a}$ have been deposited in the Cambridge Crystallographic Data Centre, CCDC No. 957068-957069. Copies of this information may be obtained free of charge from the director, CCDC, 12 Union Road, Cambridge, CB2 1EZ, U.K. (fax: 44-1223336033, email: deposit@ccdc.cam.ac.uk or http://www.ccdc.cam.ac. uk).

\section{Acknowledgements}

This work was supported by Hefei University of Technology. The authors acknowledge University of Science and Technology of China for providing the spectral measurements conditions.

\section{References}

1. (a) Guo X Q and Schulz Rolf C 1994 Polym. Int. 34 229; (b) Ikeda S -I, Kondo H, Arii T and Odashima K 2002 Chem. Commun. 202422

2. Wipf P and Wang X D 2002 J. Comb. Chem. 4656

3. Crosignani, S, Young Abigail C and Linclau B 2004 Tetrahedron. Lett. 459611

4. Schwekendiek K and Glorius F 2006 Synthesis $\mathbf{1 8} 2996$

5. Moghadam M, Mirkhani V, Tangestaninejad S, Mohammadpoor-Baltork I and Kargar H J 2009 Iran. Chem. Soc. 6251

6. Mohammadpoor-Baltork I, Moghadam M, Tangestaninejad S, Mirkhani V and Hojati S F 2008 Catal. Commun. 9 1153

7. Sheldrick G M SHELXS-97 Program for X-ray Crystal Structure Solution Göttingen University: Germany 1997; Sheldrick G M SHELXL-97 Program for Xray Crystal Structure Refinement Göttingen University: Germany 1997

8. Sout G H Jensen L H 1968 In X-ray Structure Determination: A Practical Guide (New York: MacMillan) 